\title{
Nasal Polyposis: More than a Chronic Inflammatory Disorder-A Disease of Mechanical Dysfunction- The São Paulo Position
}

\author{
Rogerio Pezato ${ }^{10}$ Richard Louis Voegels ${ }^{2}$ Shirley Pignatari ${ }^{1} \quad$ Luiz Carlos Gregório ${ }^{1}$ \\ Thiago Freire Pinto Bezerra ${ }^{3}$ Luciano Gregorio ${ }^{1}$ Leonardo Balsalobre ${ }^{1}$ Miguel Soares Tepedino ${ }^{4}$ \\ Nathália Coronel ${ }^{1}$ Fabio de Rezende Pinna ${ }^{2}$ José Mendes Neto ${ }^{1}$ Pedro Oliveira ${ }^{1}$ Eduardo Macoto ${ }^{1}$ \\ Renato Stefanini ${ }^{1}$ Claudia Figueiredo ${ }^{1}$ Fernanda Haddad ${ }^{1}$ Renata Pilan ${ }^{2}$ Ana Bezerra Soter ${ }^{2}$ \\ Nelson Almeida Melo ${ }^{2}$ Danilo Almeida Candido ${ }^{5}$ jonatas do Amaral ${ }^{1}$ Rodrigo de Paula Santos ${ }^{1}$ \\ Thibaut Van Zele ${ }^{6}$ Reginaldo Fujita ${ }^{1}$ Juliana L. Dreyfuss ${ }^{7}$ Wallace Chamon ${ }^{8,9}$ \\ Adriano Mesquita Alencar ${ }^{10}$ Claudina Perez-Novo ${ }^{11} \quad$ Aldo Cassol Stamm $^{1}$ \\ ${ }^{1}$ Department of Otolaryngology - Head and Neck Surgery, Unifesp, \\ São Paulo, SP, Brazil \\ 2 Department of Otolaryngology, USP, São Paulo, SP, Brazil \\ 3 Department of Otolaryngology, UFPE, Recife, PE, Brazil \\ ${ }^{4}$ Department of Otolaryngology, UERJ, Rio de Janeiro, RJ, Brazil \\ 5 Department of Medicine, Nephrology Division, Unifesp, São Paulo, \\ SP, Brazil \\ ${ }^{6}$ Ear, Nose and Throat Department,, University of Ghent, Ghent, Belgium \\ ${ }^{7}$ Department of Biochemistry, Unifesp, São Paulo, SP, Brazil \\ ${ }^{8}$ Department of Ophthalmology and Visual Sciences, Unifesp, São \\ Paulo, SP, Brazil \\ Address for correspondence Rogério Pezato, MD, PhD, \\ Departamento de Otorrinolaringlogia e Cirurgia de Cabeça e Pescoço, \\ Universidade Federal de São Paulo, Rua dos Otonis 700, Vila \\ Clementino,São Paulo, SP, Brazil (e-mail: pezatobau@gmail.com). \\ ${ }^{9}$ Department of Ophthalmology and Visual Sciences, College of \\ Medicine, University of Illinois at Chicago, Chicago, IL, \\ United States \\ 10 Laboratory of Microrheology and Molecular Physiology, Institute of \\ Physics, USP, São Paulo, SP, Brazil \\ 11 Proteinscience, Proteomics and Epigenetic Signaling, Department \\ of Biomedical Sciences, University of Antwerp, Antwerp, Belgium
}

Int Arch Otorhinolaryngol 2019;23:241-249.

\begin{abstract}
Keywords

- airway

- inflammation

- nasal mucosa

- nasal polyps

- sinusitis

Introduction The importance of our study lies in the fact that we have demonstrated the occurrence of mechanical dysfunction within polypoid tissues, which promotes the development of polyps in the nasal cavity.

Objective To change the paradigm of nasal polyposis (NP). In this new conception, the chronic nasal inflammatory process that occurs in response to allergies, to pollution, to changes in the epithelial barrier, or to other factors is merely the trigger of the development of the disease in individuals with a genetic predisposition to an abnormal tissue remodeling process, which leads to a derangement of the mechanical properties of the nasal mucosa and, consequently, allows it to grow unchecked.

Data Synthesis We propose a fundamentally new approach to intervening in the pathological process of NP, addressing biomechanical properties, fluid dynamics, and the concept of surface tension.

Conclusion The incorporation of biomechanical knowledge into our understanding of NP provides a new perspective to help elucidate the physiology and the pathology of nasal polyps, and new avenues for the treatment and cure of NP.
\end{abstract}

(D) Rogerio Pezato's ORCID is https://orcid.org/0000-0002-98134466.

received

May 29, 2018

accepted

October 21, 2018

published online

March 1, 2019
DOI https://doi.org/

10.1055/s-0038-1676659.

ISSN 1809-9777.
Copyright $(2019$ by Thieme Revinter

Publicações Ltda, Rio de Janeiro, Brazil
License terms

(c) $(1) \$$ 

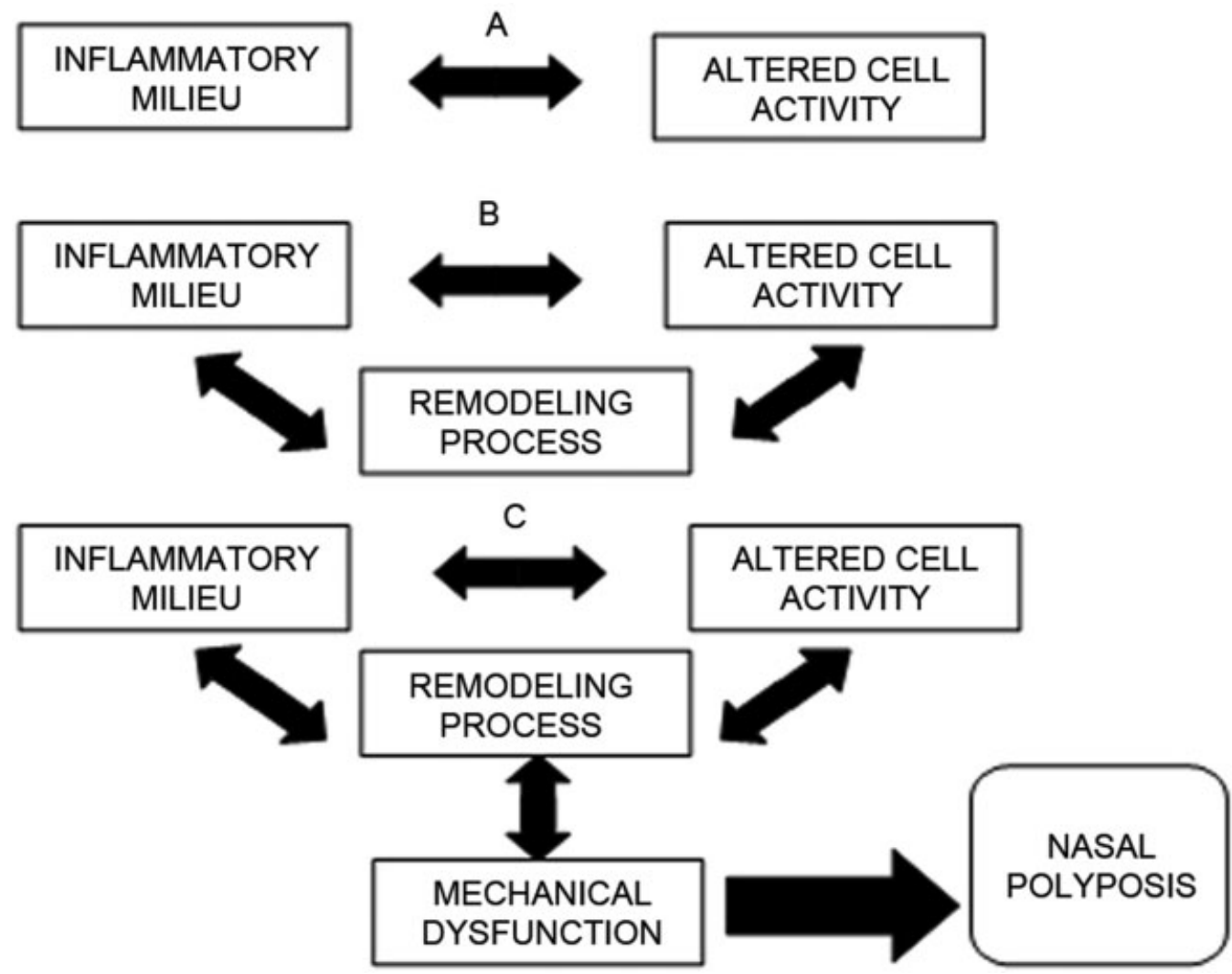

Fig. 1 Interactions for the development of nasal polyposis. 


\section{Introduction}

Nasal polyposis (NP) is essentially understood as a chronic inflammatory disease. Accordingly, the vast majority of the studies on NP have sought to understand what leads to this continuous state of inflammation.

Theories such as changes in the nasal mucosal barrier (ciliary dyskinesia, cystic fibrosis), allergy, presence of microorganisms (such as fungi and bacteria), production of superantigens, and viral infection have been proposed to explain the continuous stimulation of the inflammatory process. $^{1}$

It has also been demonstrated that patients with sensitivity to aspirin experience a chronic state of systemic inflammation due to an imbalance in the production of eicosanoids. ${ }^{2}$ In these patients, NP carries a worse prognosis.

With a focus on the inflammatory process, many studies have implicated functional abnormalities of various cell types -such as macrophages, dendritic cells, and stem cells-in the maintenance of the inflammation, ${ }^{3-9}$ while other studies have demonstrated the effect of the inflammatory milieu on cell activity and differentiation, especially in Th2-driven NP. ${ }^{10-12}$

As a result of these extensive efforts to understand the inflammatory process of NP, hundreds of mediators of inflammation have been implicated in its development.

Changes in the extracellular environment have been found to influence cell activity, while intrinsic changes in the cells, in turn, alter the environment to which they are exposed ( $\mathbf{F i g}$. 1A).

Evolving concepts of the chronic inflammatory process in $\mathrm{NP}$ have revealed its intimate relationship with the remodeling process and its reciprocal relationship with the amplification of inflammation, with particular involvement of substances produced by metalloproteinases ${ }^{13,14}$ ( - Fig. 1B).

Despite the wealth of knowledge generated by research on the inflammatory process in NP, there has been little impact on the management of the disease; its treatment still relies on anti-inflammatory drugs (especially corticosteroids) and surgical resection.

The purpose of the present manifesto is to introduce concepts of mechanical processes that occur in NP ( - Fig. 1C) and their implications in understanding this disease.

But first, we must review some facts that will help us shift our interest away from the inflammatory process in NP and encourage us to investigate new aspects of this disease.

In 2008, an article by Zhang et al made it clear that the inflammatory process of NP is not unique to this condition. The authors showed that NP is not simply a disease orchestrated by Th2 cells, as is predominant in the West; there is also a high prevalence of NP orchestrated by non-Th2 T

Table 1 Differences between nasal and lung mucosa

\begin{tabular}{|l|}
\hline Vascular system and its permeability \\
\hline Remodeling process and interstitial matrix \\
\hline Role of cells not found in the nasal mucosa, such as \\
smooth muscle cells, myofibroblasts, chondrocytes \\
\hline
\end{tabular}

Table 2 Important concepts in the mechanical dysfunction of nasal polyposis

\begin{tabular}{|l|}
\hline Young-Laplace law \\
\hline Pressure imbalance \\
\hline $\begin{array}{l}\text { Differences between the mechanical properties of the } \\
\text { submucosa in the inferior turbinate and in the middle } \\
\text { turbinate set them apart }\end{array}$ \\
\hline Vascularization \\
\hline Cellular traction force \\
\hline
\end{tabular}

helper cells, especially in Asia. ${ }^{15}$ Therefore, any inflammatory process may be involved in the development of NP, independent of the predominant $\mathrm{T}$ cell subtype or polarization. These findings have been corroborated extensively by subsequent Asian studies. ${ }^{16,17}$

These findings suggest that we must either individualize these distinct inflammatory processes before attempting to treat them, or, instead, that we should address the problem by preventing the consequences of inflammation in the nasal mucosa affected by NP, regardless of the type of inflammatory process present.

The latter option is particularly tempting because of the challenges of subclassifying the inflammatory process and the impossibility of avoiding it altogether, since the nasal mucosa is perennially exposed to external insults such as allergens, pollution, viruses, bacteria, and fungi.

Another concept that drives us to study mechanical dysfunction in NP is the fact that polyposis does not occur in the bronchial mucosa, although it is lined by the same epithelium as the upper airway (pseudostratified columnar epithelium), and many authors consider the upper and lower airways a single structure ("one airway, one disease" concept).

Unlike NP, bronchial polyposis could have led to an incredible disadvantage in the natural selection, causing this mucosa to develop a series of protective factors that inhibit polyp growth in the lower airway, despite the exposure to the same exogenous aggressors as in the upper region of the airway, making it eligible as a model for understanding the development of nasal polyps. ${ }^{18}$ This led us to begin an indepth study of the main differences between the regions of the airway (-Table $\mathbf{1}$ ).

The same study that suggested the use of the bronchial mucosa as a model for studies on NP also raised the hypothesis that the nasal mucosa presented less resistance to edema formation in NP..$^{18}$ Despite the fact that the polypoid nasal mucosa is empirically recognized as a soft tissue, and that the nasal mucosa in chronic sinusitis without nasal polyposis is empirically recognized as a fibrotic tissue, only in 2014 it was confirmed for the first time that the nasal mucosa of patients with NP is more conducive to edema formation through biomechanical dysfunction. ${ }^{19}$ This same study demonstrated that not only the polypoid tissue, but the entire nasal mucosa of patients with NP exhibits a form of mechanical dysfunction, and that this dysfunction varies according to the region of the nasal mucosa. 
Subsequent biomechanical studies have shown that a healthy nasal mucosa also exhibits different mechanical properties depending on the region, which provides further evidence against the use of the lower-turbinate mucosa as a control tissue in studies of middle meatus diseases, such as NP. ${ }^{20}$

- Table 2 summarizes important biomechanical concepts that may be implicated in the development of NP.

\section{Review on the Properties of Nasal Polyposis}

\section{Fluid Dynamics and Surface Tension}

The mechanical behavior of soft materials, especially when they interface with fluids, is a recurrent question in biology and medicine, ${ }^{21}$ attracting researchers from different fields. However, in any field, ${ }^{22,23}$ the Young-Laplace equation is recognized as perhaps the most classical observation. ${ }^{24}$ The Young-Laplace equation relates curvature, pressure, and tension across the interface between two fluids, such as water and air. It can also be used to describe the wall tension across a thin membrane that separates two fluids or a fluid and a soft material. ${ }^{22}$

If a pressure difference $(\Delta P)$ exists across a thin membrane separating two fluids, the shape of this membrane is curved and, at any point of it, one can define a radius of curvature (R). In this case, the membrane has to be strong enough to sustain this pressure difference. It is interesting to highlight that, even for the same $\Delta P$, the tension on the membrane, or wall tension $\left(T_{\mathrm{W}}\right)$, will change if the curvature of the membrane changes. The Young-Laplace equation relates these quantities, and for spherical curvatures, it is given by Eq. 1, shown below:

$$
T_{\mathrm{W}}=\frac{\Delta P R}{2}(1)
$$

Thus, the larger the radius of the curvature $(R)$ the greater its wall tension $\left(T_{\mathrm{W}}\right)$, Thus, one may conclude that structures with a smaller $R$ will be able to tolerate greater pressure differences $(\Delta P)$.

This is one of the reasons why filling a balloon requires considerable effort initially and less effort as the balloon inflates. At any time, the balloon has a radius $R$, and to inflate the balloon, one has to increase the internal pressure, which also increases the $\Delta P$, since the $\Delta P$ is the pressure difference across the balloon membrane; in other words, the internal pressure minus the atmospheric pressure. Once a $\Delta P$ exists, the membrane of the balloon tends to expand until the wall tension $T_{\mathrm{W}}$ is strong enough to overcome the forces caused by the $\Delta P$. Thus, at the beginning, a small $R$ requires a greater $\Delta P$, and, consequently, a greater effort, than for a bigger $R$, when the balloon is full (see Eq. 1). Eventually, the $T_{\mathrm{W}}$ reaches the maximum tension supported by the membrane, which will break it.

The same concept can be applied to the tension in vessel walls. Small aneurysms have a lower risk of rupture, because, for the same $\Delta P$, the wall tension $T_{\mathrm{W}}$ required is smaller. This effect also leads to a slower growth because their smaller diameter leads to less tension in the wall; consequently, in larger aneurysms, the tensile force at the wall is greater because of the increased $R$, and small changes in pressure greatly increase the tension at the vessel walls, increasing the risk of rupture.
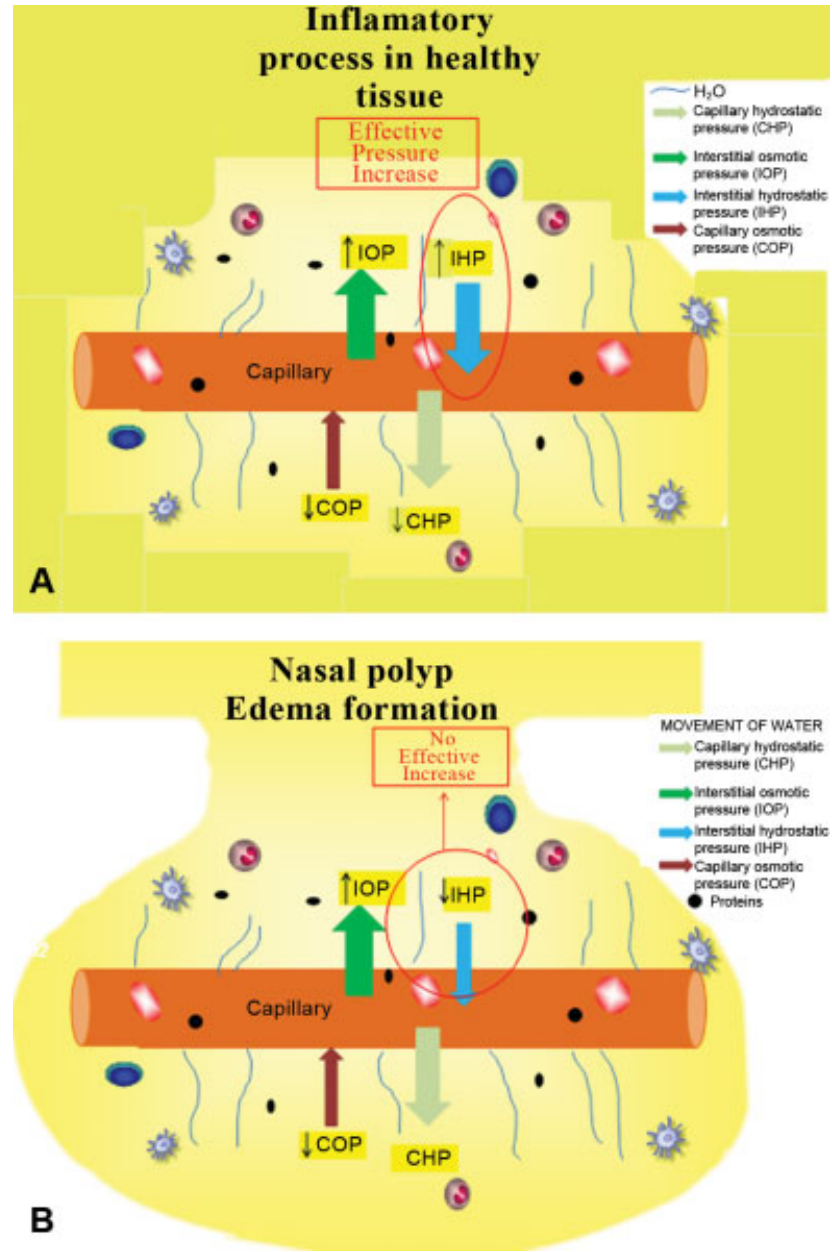

Fig. 2 (A) Scheme showing the balance between hydrostatic and oncotic pressure during the inflammatory process in a healthy nasal mucosa. (B) Scheme showing an impaired increase of interstitial hydrostatic pressure in the nasal polypoid mucosa, during the inflammation process, unable to counterbalance the increase of the interstitial osmotic pressure. ${ }^{19}$

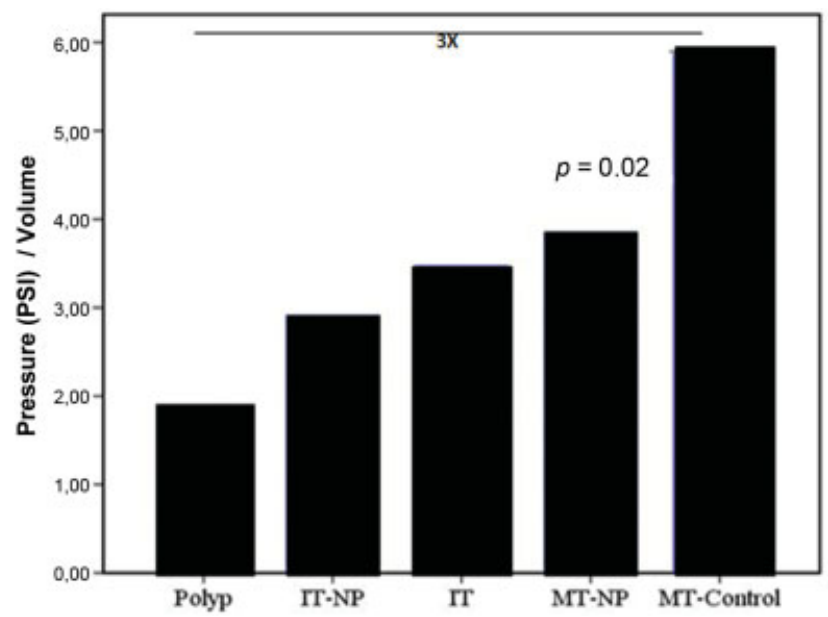

Fig. 3 Graphic of the increase of pressure per $0.1 \mathrm{ml}$ added into the nasal mucosa. It is shown that the middle meatus mucosa from controls has a three times higher interstitial hydrostatic pressure response than the polypoid mucosa ${ }^{19}$ Abbreviations: IT, inferior turbinate; MT, middle turbinate; NP, nasal polyposis. 
Another important concept here was found after the landmark experiments of Torricelli. The experiments demonstrated that, when a fluid is confined between the walls of one material and interfaces with another fluid, such as air, the shapes of both fluids change. The new shape depends on the material properties of both fluids, as well as on the material properties of the confining wall and its diameter.

\section{Pressure Imbalance and Edema Formation}

Within the context of the physical properties of polypoid nasal tissue, an important mechanism that facilitates altered remodeling is the development of cumulative edema in the polyp.

Water may be either bound to the extracellular matrix or free in the interstitial tissue.

Due to a breakdown in the balance of pressure forces that govern the movement of water between the capillaries and the tissues, the NP milieu facilitates the outflow of water from the capillaries and its buildup in the interstitial space, contributing to the development of edema.

To maintain equilibrium in the flow of water between the tissue and the capillaries, the sum of the forces acting toward the outflow of water from the capillaries (hydrostatic pressure in the capillaries + osmotic pressure in the surrounding tissue) tends to be equal to the sum of the forces acting toward the inflow of water from the tissue to the capillaries (hydrostatic pressure in the interstitial space + osmotic pressure in the capillaries), thus preventing edema from developing (-Fig. 2).
Usually, the hydrostatic pressure vector Ph (difference between the hydrostatic pressure in the capillary and the hydrostatic pressure in the tissue) tends to equalize with the oncotic pressure vector Po (difference between the osmotic pressure in the capillary and in the tissue), acting in the opposite direction: $[\mathrm{Ph}]=[\mathrm{Po}]$.

In a healthy tissue, during an inflammatory process, proteins are lost from the capillaries to the interstitial space due to the increased permeability and, consequently, exudation and increased osmotic pressure in the interstitial space. Simultaneously with the increased outflow of water to the interstitial space due to the increase of the osmotic pressure therein, the hydrostatic pressure in the interstitial space rises and the hydrostatic pressure in the capillaries drops due to hypovolemia. This mechanism plays an essential role in limiting edema and preventing unchecked volume loss, which would lead to tissue rupture or hypovolemic shock.

Recent experimental studies have shown that, in NP, the increase in the interstitial hydrostatic pressure in response to volume extravasation is inadequate, requiring a 3-fold greater volume of fluid to reach the same hydrostatic pressure of the nasal mucosa of the healthy middle meatus ${ }^{19,20}$ (-Fig. 3 ).

Another important finding of these studies is that, in healthy individuals, the middle meatus mucosa exhibits the greatest interstitial hydrostatic pressure response to volume extravasation - a response that is seriously compromised in NP.

This extremely efficient interstitial hydrostatic pressure response of the normal mucous membrane of the middle

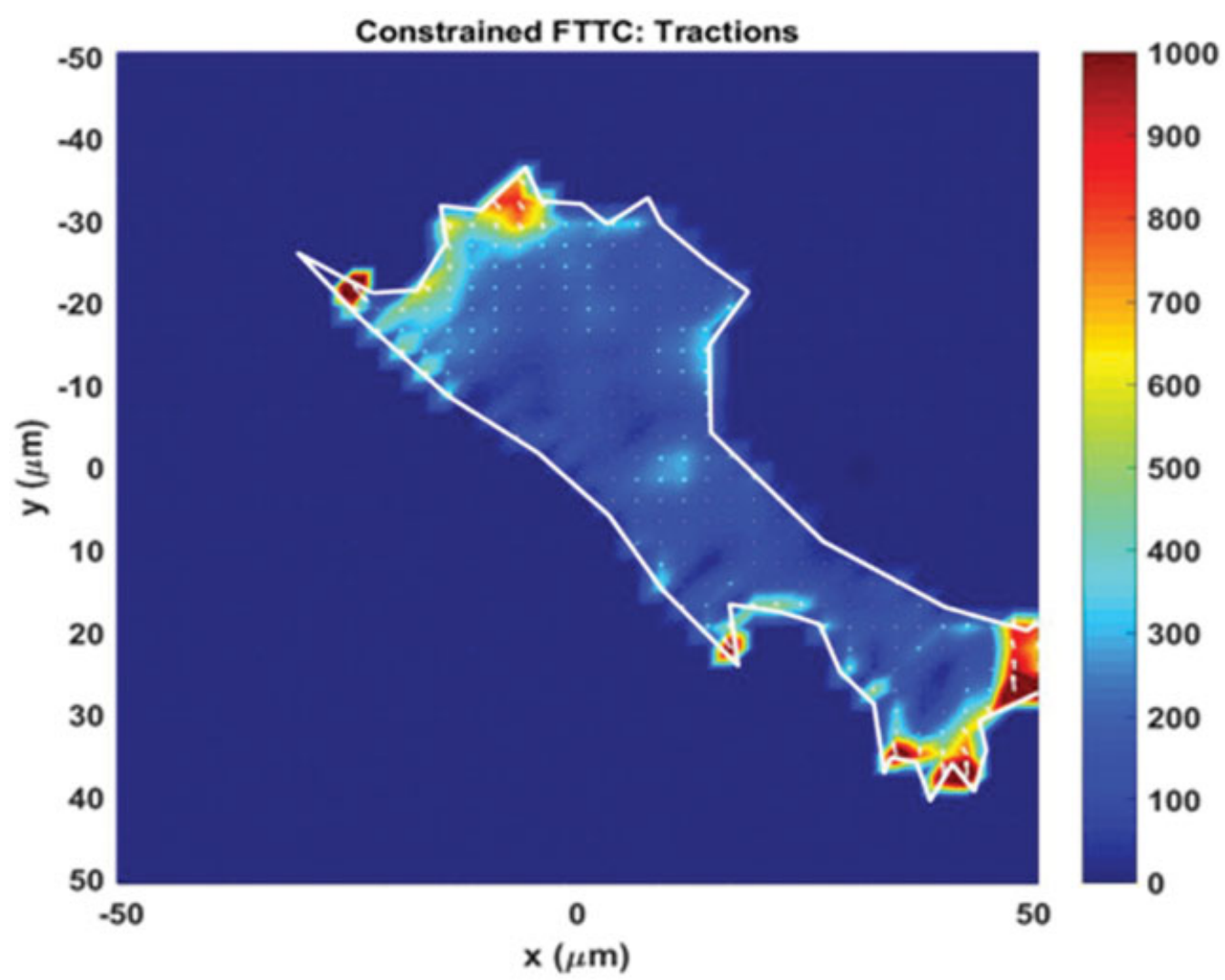

Fig. 4 Illustration of the mechanical forces exerted by the fibroblast from the nasal mucosa by Fourier-transform traction microscopy. Abbreviation: FTTC: Fourier-transform traction microscopy. 
meatus, as a compensatory factor, is closely related to the unique characteristics developed by the extracellular matrix that composes this region ${ }^{25}$ to face the equally peculiar microenvironmental features of this region. ${ }^{26-29}$

Again, this concept integrates our understanding of the dyad of chronic inflammation and mechanical dysfunction in $\mathrm{NP}$, whereby the inflammatory process maintains oncotic pressure chronically at a level that facilitates volume loss from the capillaries to the surrounding tissue, while mechanical dysfunction hinders the return of this lost fluid to the vasculature. This creates an environment conducive to altered remodeling and establishes a negative feedback loop that potentiates the failure of interstitial hydrostatic pressure control in response to volume extravasation into the interstitial space.

\section{Different Mechanical Properties of Different Anatomical Regions in the Nose}

It has long been known that the nasal mucosa of the middle meatus has histological, metabolic, and airflow differences from the nasal mucosa found in the inferior turbinate. ${ }^{22-26}$ Biomechanical studies have demonstrated that these structures have different mechanical properties as well. ${ }^{19,20}$ In a healthy nasal mucosa, the middle meatus exhibits the greatest increase in interstitial hydrostatic pressure in response to fluid infusion. This is a pivotal mechanism for the inhibition of nasal polyp growth in this region, which, as we will demonstrate with the concept of three-way contact, is singularly susceptible to polyp development because it is the narrowest portion of the outlets created by the nasal turbinates.

In NP, the mucosal tissue loses its responsiveness. The increase in the hydrostatic pressure in NP-affected mucosa is three times lower than the increase in the interstitial hydrostatic pressure of a healthy middle meatus mucosa for the same infused volume of fluid (-Fig. 3 ).

Biomechanical studies of the nasal mucosa have also shown that patients with NP exhibit these mechanical abnormalities not only in the polypoid mucosa, but also in the middle meatus and in the inferior turbinates, which suggests that the disease is disseminated throughout the nasal mucosa (-Fig. 3 ) rather than limited to the middle meatus.

\section{Cellular Traction Force}

Adherent cells such as epithelium, fibroblast, and stem cells produce cellular traction force on a substrate to keep their physical morphologies, to sense the external environment, and to perform essential cellular functions. ${ }^{30}$

Thus, the adherent cells themselves also contribute to the tissue biomechanical properties, the way that they are distributed in the tissue, the density and proportion of these cells, result in a traction force applied in different environment. This cellular traction force field can be measured, in vitro, by Fourier-transform traction microscopy, ${ }^{31}$ as shown in - Fig. 4.

\section{Discussion on the Clinical Implications of Mechanical Dysfunction in Nasal Polyposis}

\section{Fluid Mechanics}

The application of the tenets of physics to this new knowledge of the biomechanical properties of nasal polyp tissue can enhance our understanding and, especially, improve our management of NP.

The Young-Laplace equation allows us to characterize polypoid tissue as a droplet-like structure, which generates

\section{Nasal polyp}

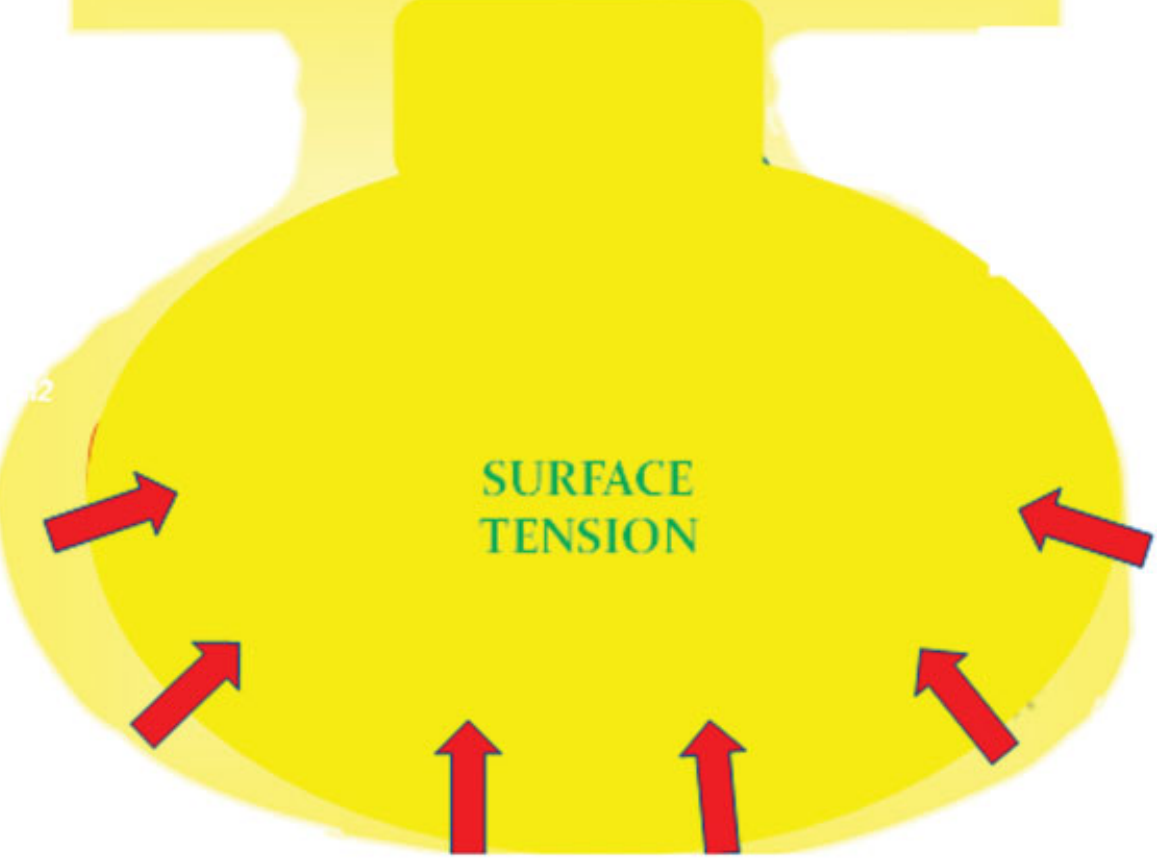

Fig. 5 Illustration of surface tension response to balance with the internal polyp pressure. The surface tension is related to the diameter of the polyp. 
a wall tension $\left(T_{\mathrm{W}}\right)$ at its interface with air. This tension prevents the tissue from bursting, since the pressure within the polyp is greater than the air pressure acting upon it, leading to a deformation known as a meniscus in the polypoid tissue.

To calculate the $T_{\mathrm{W}}$, the Eq. (1) is used, where $R$ is the radius of the meniscus and $\Delta P$ is the pressure difference between the inside and the outside of the polyp.

Thus, in our model, the interface region polyp-air and also the interface polyp-wall exerts a wall tension in the interior of the polyp and differs according to its size.

This model provides a partial explanation for polyp growth, where polyps with increased radius require an increased wall tension (-Fig. 5).

Another important finding drawn from fluid biophysics is that, in its initial stages, when it is still small, a polyp will tolerate a much higher pressure increase with little deformation, because it generates a small fluctuation in the wall tension. As it grows, smaller increases in the internal pressure within the polyp will lead to significant increases in the wall tension at the expense of further growth, which demonstrates the importance of keeping polyps physically small, because their rate of growth increases as their diameter increases.

Many authors believe that the contact between two nasal mucous membranes would facilitate the development of polyposis, especially due to a failure of effective ciliary motion and to the generation of an inflammatory process in the area of contact.

The principles of fluid mechanics can partially explain the changes that occur on the surface of the polyp during three- way contact, as well as explain why the middle meatus is the predominantly affected area.

As noted above, a polyp acts as a fluid component with fluid characteristics within our system and is in contact with air. As it grows, the polyp comes into contact with a third, more solid medium (epithelium-lined bone or cartilage). In contact with a solid medium, the polyp grows toward the air, due to the lower resistance of this medium.

During the contact among these three media, the convexity of the meniscus may be increased, and the $T_{\mathrm{W}}$ may be altered.

Finally, acting on the surface tension in the epithelial cells of the polyp can decisively alter their communication with stromal cells and, consequently, affect the remodeling process, which suggests a potential for applicability of substances that interfere with surface tension.

\section{Pressure Imbalance in Nasal Polyposis}

As shown above, the difficulty in raising the interstitial hydrostatic pressure during inflammation in a polyposisaffected nasal mucosa is conducive to the development of edema and may be implicated in the remodeling process.

This points to a new therapeutic avenue that should be pursued: the elevation of the interstitial hydrostatic pressure regardless of the type of inflammatory process involved, that is, seeking to treat not the cause of the disease but its terminal clinical manifestation, which is the same for different types of NP (Th1-driven, Th2-driven, and NP due to cystic fibrosis).

In an analogy with nature, the genetic factors that predispose to NP would correspond to the source of a river; the

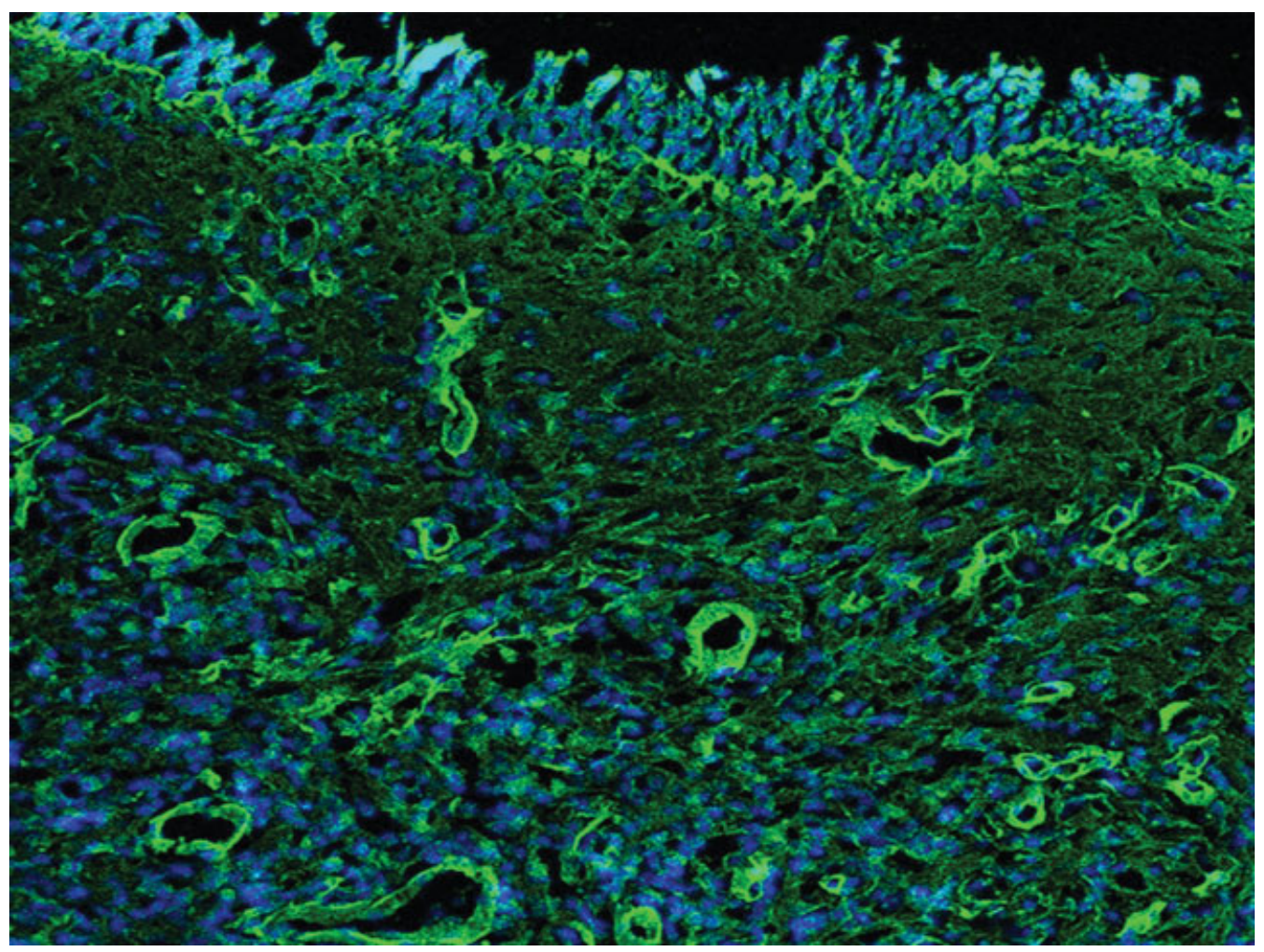

Fig. 6 Immunohistochemistry after crosslinking process, nasal mucosa tissue. In green, collagen type I. 
chronic inflammatory process would correspond to the rains, thaws, and tributaries that cause the water level of this river to rise; and the nasal polyps themselves would correspond to flash floods, causing symptoms in the patient and catastrophic damage to the population.

Much research has been conducted on the genetic and epigenetic factors of NP, but little has been discovered or found to be useful in terms of disease markers or treatment targets.

The majority of this research has focused on establishing the type of inflammatory process, on investigating environmental factors that enhance the inflammatory process, and on the role of major inflammatory cells in NP. All of the current clinical treatment approaches are based on this knowledge (use of corticosteroids, anti-immunoglobulin E [IgE] agents, leukotriene receptor blockers, macrolides, antiinterleukin 5 [IL-5] agents, antihistamines, etc.). ${ }^{1,32-36}$

The biomechanical knowledge presented in this manifesto would allow new approaches to focus instead on the effects of the inflammatory process in a tissue predisposed to the development of polyposis.

Continuing with our analogy, we would be acting downstream of the source of the river, widening the river bed and improving water drainage systems, with no concern for any rains, thaws, or tributaries.

Since the interstitial hydrostatic pressure response is related to the extracellular matrix and polypoid tissue is characterized by loose tissue with low collagen content, ${ }^{37}$ while chronic sinusitis without polyposis is characterized by fibrous tissue, it is interesting from a biomechanical standpoint to evaluate the interstitial hydrostatic pressure response in fibrotic nasal tissue.

In one study, the interstitial tissue hydrostatic pressure response in nasal tissue with fibrosis (synechiae) was compared with that of polypoid tissue. Fibrotic tissue was found to be biomechanically similar to normal nasal tissue, ${ }^{38}$ paving the way for the treatment of NP by manipulating the quantity and quality of the extracellular matrix.

Several substances may potentially act as sclerosing or degrading agents to alter the composition of the extracellular matrix and may potentially act on the interstitial hydrostatic pressure. However, the extracellular matrix can also be modified qualitatively.

Crosslinking is a natural process that occurs with tissue aging and is also used industrially to increase the strength of materials through polymer bonding. In medicine, crosslinking has been applied to the cornea of patients with keratoconus to stabilize the progression of corneal bulging. ${ }^{39}$

To change the properties of the extracellular matrix in the nasal mucosa, it is possible to apply the crosslinking process (-Fig. 6), which makes this process a promising alternative to stiffen the scaffold of the nasal mucosa and to prevent the development of NP in predisposed patients.

The interstitial hydrostatic pressure can also be increased by transmission of external pressure. To evaluate the accuracy of the pressure-response imbalance theory of the pathogenesis of NP, in one study, patients with NP were acutely exposed to continuous positive pressure in the nasal cavity. Surprisingly, the nasal polyp volume was reduced ${ }^{40}$, probably due to the successful transmission of continuous pressure to the affected tissue, raising the interstitial hydrostatic pressure and consequently facilitating the flow of water back into the capillaries.

\section{Anatomical Regions in the Nose}

One particularly important contribution of the research on mechanical dysfunction in NP is the demonstration that the entire nasal mucosa is affected, not only the middle meatus or the polyp tissue itself. ${ }^{19,20}$

However, the greatest contribution of this line of research may lie in the finding that the mechanical properties of the nasal mucosa differ between the different anatomical regions of the nose, both in healthy individuals and in patients with NP. This has revealed a clear methodological failure in previous studies that used the lower turbinate mucosa as a control for NP tissue, since these two mucous membranes cannot be compared. ${ }^{20}$

A greater methodological rigor in future researches is highly recommended to obtain more reliable responses and to reduce conflicting findings, thus avoiding the publication of misleading data.

It is not scientifically acceptable to compare polypoid tissues with any other nasal mucosa not originating from the middle meatus.

\section{Final Comments}

The incorporation of biomechanical knowledge into our understanding of NP provides a new perspective to help elucidate the physiology and the pathology of nasal polyps, as well as new avenues for the treatment and cure of NP.

Conflicts of Interest

The authors have no conflicts of interest to declare.

\section{References}

1 Fokkens WJ, Lund VJ, Mullol J, et al. EPOS 2012: European position paper on rhinosinusitis and nasal polyps 2012. A summary for otorhinolaryngologists. Rhinology 2012;50(01):1-12. Doi: 10.4193/ Rhino50E2

2 Pezato R, Świerczyńska-Krępa M, Niżankowska-Mogilnicka E, Derycke L, Bachert C, Pérez-Novo CA. Role of imbalance of eicosanoid pathways and staphylococcal superantigens in chronic rhinosinusitis. Allergy 2012;67(11):1347-1356. Doi: 10.1111/all.12010

3 Poposki JA, Peterson S, Welch K, et al. Elevated presence of myeloid dendritic cells in nasal polyps of patients with chronic rhinosinusitis. Clin Exp Allergy 2015;45(02):384-393. Doi: $10.1111 /$ cea. 12471

4 Pezato R, Pérez-Novo CA, Holtappels G, et al. The expression of dendritic cell subsets in severe chronic rhinosinusitis with nasal polyps is altered. Immunobiology 2014;219(09):729-736. Doi: 10.1016/j.imbio.2014.05.004

5 Van Bruaene N, Pérez-Novo CA, Basinski TM, et al. T-cell regulation in chronic paranasal sinus disease. J Allergy Clin Immunol 2008;121 (06):1435-1441, 1441.e1-1441.e3. Doi: 10.1016/j.jaci.2008.02.018 
6 Perez-Novo C, Pezato R. Dendritic cell subset expression in severe chronic rhinosinusitis with nasal polyps. Curr Opin Allergy Clin Immunol 2017;17(01):1-4. Doi: 10.1097/ACI.0000000000000328

7 Pezato R, de Almeida DC, Bezerra TF, et al. Immunoregulatory effects of bone marrow-derived mesenchymal stem cells in the nasal polyp microenvironment. Mediators Inflamm 2014; 2014:583409. Doi: $10.1155 / 2014 / 583409$

8 de Oliveira PWB, Pezato R, Agudelo JSH, et al. Nasal Polyp-Derived Mesenchymal Stromal Cells Exhibit Lack of Immune-Associated Molecules and High Levels of Stem/Progenitor Cells Markers. Front Immunol 2017;8:39. Doi: 10.3389/fimmu.2017.00039

9 Krysko O, Vandenabeele P, Krysko DV, Bachert C. Impairment of phagocytosis of apoptotic cells and its role in chronic airway diseases. Apoptosis 2010;15(09):1137-1146. Doi: 10.1007/s10495-010-0504-X

10 Lam EPS, Kariyawasam HH, Rana BMJ, et al. IL-25/IL-33-responsive TH2 cells characterize nasal polyps with a default TH17 signature in nasal mucosa. J Allergy Clin Immunol 2016;137 (05):1514-1524. Doi: 10.1016/j.jaci.2015.10.019

11 Dilidaer ZY, Zheng Y, Liu Z, et al. Increased BAFF expression in nasal polyps is associated with local IgE production, Th2 response and concomitant asthma. Eur Arch Otorhinolaryngol 2017;274 (04):1883-1890. Doi: 10.1007/s00405-016-4435-1

12 Lan F, Wang XD, Nauwynck HJ, et al. Th2 biased upper airway inflammation is associated with an impaired response to viral infection with Herpes simplex virus 1. Rhinology 2016;54(02): 141-149. Doi: 10.4193/Rhin15.213

13 Balsalobre L, Pezato R, Perez-Novo C, et al. Epithelium and stroma from nasal polyp mucosa exhibits inverse expression of TGF- $\beta 1$ as compared with healthy nasal mucosa. J Otolaryngol Head Neck Surg 2013;42(APR):29. Doi: 10.1186/1916-0216-42-29

14 Pezato R, Balsalobre L, Lima M, et al. Convergence of two major pathophysiologic mechanisms in nasal polyposis: immune response to Staphylococcus aureus and airway remodeling. J Otolaryngol Head Neck Surg 2013;42(01):27. Doi: 10.1186/1916-0216-42-27

15 Zhang N, Van Zele T, Perez-Novo C, et al. Different types of Teffector cells orchestrate mucosal inflammation in chronic sinus disease. J Allergy Clin Immunol 2008;122(05):961-968. Doi: 10.1016/j.jaci.2008.07.008

16 Wang $X$, Zhang N, Bo M, et al. Diversity of $T_{H}$ cytokine profiles in patients with chronic rhinosinusitis: A multicenter study in Europe, Asia, and Oceania. J Allergy Clin Immunol 2016;138 (05):1344-1353. Doi: 10.1016/j.jaci.2016.05.041

17 Shin S-H, Ye M-K, Kim J-K, Cho C-H. Histological characteristics of chronic rhinosinusitis with nasal polyps: Recent 10-year experience of a single center in Daegu, Korea. Am J Rhinol Allergy 2014; 28(02):95-98. Doi: 10.2500/ajra.2014.28.4003

18 Pezato R, Voegels RL. Why do we not find polyps in the lungs? Bronchial mucosa as a model in the treatment of polyposis. Med Hypotheses 2012;78(04):468-470. Doi: 10.1016/j.mehy.2012.01.006

19 Pezato R, Voegels RL, Pinto Bezerra TF, Perez-Novo C, Stamm AC, Gregorio LC. Mechanical disfunction in the mucosal oedema formation of patients with nasal polyps. Rhinology 2014;52 (02):162-166. Doi: $10.4193 /$ Rhin 13.066

20 Pezato R, Voegels RL, Stamm AC, Gregorio LC. Why we should avoid using inferior turbinate tissue as control to Nasal Polyposis studies. Acta Otolaryngol 2016. doi: 10.3109

21 Alencar AM, Ferraz MS, Park CY, et al. Non-equilibrium cytoquake dynamics in cytoskeletal remodeling and stabilization. Soft Matter 2016;12(41):8506-8511. Doi: 10.1039/c6sm01041e

22 Almeida AB, Giovambattista N, Buldyrev SV, Alencar AM. Validation of Capillarity Theory at the Nanometer Scale. II: Stability and Rupture of Water Capillary Bridges in Contact with Hydrophobic and Hydrophilic Surfaces. J Phys Chem C 2018;122(03):1556-1569
23 Style RW, Jagota A, Hui CY, Dufresne ER. Elastocapillarity. . Surface Tensionand the Mechanics of Soft SolidsAnnual Review of Condensed Matter Physics. 2017;8:99-118

24 Hauksbee F. An account of an experiment touching the direction of a drop of oil of oranges, between two glass planes, towards any side of them that is nearest press'd together. Philos Trans R Soc Lond 1711;27:374-375

25 Berger G, Finkelstein Y, Ophir D, Landsberg R. Old and new aspects of middle turbinate histopathology. Otolaryngol Head Neck Surg 2009;140(01):48-54. Doi: 10.1016/j.otohns.2008.10.006

26 Wang Y, Lee HP, Gordon BR. Impacts of fluid dynamics simulation in study of nasal airflow physiology and pathophysiology in realistic human three-dimensional nose models. Clin Exp Otorhinolaryngol 2012;5(04):181-187. Doi: 10.3342/ceo.2012.5.4.181

27 Takeno S, Yoshimura H, Kubota K, Taruya T, Ishino T, Hirakawa K. Comparison of nasal nitric oxide levels between the inferior turbinate surface and the middle meatus in patients with symptomatic allergic rhinitis. Allergol Int 2014;63(03):475-483. Doi: 10.2332/allergolint.14-OA-0689

28 Kaspar U, Kriegeskorte A, Schubert T, et al. The culturome of the human nose habitats reveals individual bacterial fingerprint patterns. Environ Microbiol 2016;18(07):2130-2142. Doi: 10.1111/ 1462-2920.12891

29 Biswas K, Hoggard M, Jain R, Taylor MW, Douglas RG. The nasal microbiota in health and disease: variation within and between subjects. Front Microbiol 2015;9:134. Doi: 10.3389/fmicb.2015.00134

30 Holley MT, YekrangSafakar A, Maziveyi M, Alahari SK, Park K. Measurement of cell traction force with a thin film PDMS cantilever. Biomed Microdevices 2017;19(04):97

31 Park CY, Tambe D, Alencar AM, et al. Mapping the cytoskeletal prestress. Am J Physiol Cell Physiol 2010;298(05):C1245-C1252. Doi: 10.1152/ajpcell.00417.2009

32 Penn R, Mikula S. The role of anti-IgE immunoglobulin therapy in nasal polyposis: a pilot study. Am J Rhinol $\cdots ; 21(04): 428-432$

33 Vennera MdelC, Picado C, Mullol J, Alobid I, Bernal-Sprekelsen M. Efficacy of omalizumab in the treatment of nasal polyps. Thorax 2011;66(09):824-825. Doi: 10.1136/thx.2010.152835

34 Pezato R, Claeys C, Holtappels G, Bachert C, Pérez-Novo C. LTD4 and TGF- $\beta 1$ Induce the Expression of Metalloproteinase- 1 in Chronic Rhinosinusitis via a Cysteinyl Leukotriene Receptor 1Related Mechanism. Sinusitis. 2016;1(01):65-75. Doi: 10.3390/ sinusitis1010065

35 Gevaert P, Lang-Loidolt D, Lackner A, et al. Nasal IL-5 levels determine the response to anti-IL-5 treatment in patients with nasal polyps. J Allergy Clin Immunol 2006;118(05):1133-1141. Doi: $10.1016 /$ j.jaci.2006.05.031

36 Haye R, Aanesen JP, Burtin B, Donnelly F, Duby C. The effect of cetirizine on symptoms and signs of nasal polyposis. J Laryngol Otol 1998;112(11):1042-1046

37 Van Bruaene N, Derycke L, Perez-Novo CA, et al. TGF-beta signaling and collagen deposition in chronic rhinosinusitis. J Allergy Clin Immunol 2009;124(02):253-259, 259.e1-259.e2

38 Gregório L, Pezato R, Felici RS, Kosugi EM. Fibrotic Tissue and Middle Turbinate Exhibit Similar Mechanical Properties. Is Fibrosis a Solution in Nasal Polyposis? Int Arch Otorhinolaryngol 2017; 21(02):122-125

39 Bottos KM, Oliveira AG, Bersanetti PA, et al. Corneal absorption of a new riboflavin-nanostructured system for transepithelial collagen cross-linking. PLoS One 2013;8(06):e66408. Doi: 10.1371/ journal.pone.0066408

40 Balsalobre L, Pezato R, Joao MG, Gregório L, Haddad FLM, Gregorio LC, Fujita R. What is the Impact of Positive Airway Pressure in Nasal Polyposis? An Experimental Study. Int Arch Otorhinolaryngol 2018; in press. DOI: 10.1055/s-0038-1676095 